\title{
Prior Stroke in PFO Patients Is Associated With Both PFO-Related and -Unrelated Factors
}

\author{
Timo Kahles ${ }^{1}$, Patrik Michel ${ }^{2}$, Alexander Hapfelmeier ${ }^{3}$, Franz R. Eberli ${ }^{4}$, \\ Marialuisa Zedde ${ }^{5}$, Vincent Thijs ${ }^{6,7}$, Markus Kraemer ${ }^{8,9}$, Stefan T. Engelter ${ }^{10,11}$, \\ Joaquin Serena ${ }^{12}$, Christian Weimar ${ }^{13}$, Achim Mallmann ${ }^{14}$, Andreas Luft ${ }^{15}$, \\ Dimitri Hemelsoet ${ }^{16}$, David E. Thaler ${ }^{17}$, Andreas Müller-Eichelberg ${ }^{18}$, Adinda De Pauw ${ }^{19}$, \\ Roman Sztajzel ${ }^{20}$, Carmel Armon 21,22, David M. Kent ${ }^{23}$, Bernhard Meier ${ }^{24}$, \\ Heinrich P. Mattle ${ }^{25}$, Urs Fischer ${ }^{25}$, Marcel Arnold ${ }^{25}$, Marie-Luise Mono ${ }^{25 t}$ and \\ Krassen Nedeltchev ${ }^{1,25 * \dagger}$ for the International PFO Consortium NCT00859885
}

\section{OPEN ACCESS}

Edited by:

Alejandro Bustamante, Hospital Germans Trias i Pujol, Spain

Reviewed by: Georgios Tsivgoulis, National and Kapodistrian University of Athens, Greece

Stefan Greisenegger, Medical University of Vienna, Austria

*Correspondence:

Krassen Nedeltchev krassen.nedeltchev@ksa.ch

†These authors have contributed equally to this work and share senior authorship

Specialty section: This article was submitted to Stroke,

a section of the journa Frontiers in Neurology

Received: 18 March 2020 Accepted: 07 May 2020

Published: 04 June 2020

Citation:

Kahles T, Michel P, Hapfelmeier A, Eberli FR, Zedde M, Thijs V, Kraemer M, Engelter ST, Serena J,

Weimar C, Mallmann A, Luft A, Hemelsoet $D$, Thaler $D E$,

Müller-Eichelberg A, De Pauw A

Sztajzel R, Armon C, Kent DM,

Meier B, Mattle HP, Fischer U, Arnold M, Mono M-L and Nedeltchev $K$ (2020) Prior Stroke in PFO Patients Is Associated With Both PFO-Related

and -Unrelated Factors.

Front. Neurol. 11:503.

doi: 10.3389/fneur.2020.00503
${ }^{1}$ Department of Neurology, Cantonal Hospital Aarau, Aarau, Switzerland, ${ }^{2}$ Department of Neurology, University Hospital of Lausanne (CHUV), Lausanne, Switzerland, ${ }^{3}$ Institute of Medical Informatics, Statistics and Epidemiology, School of Medicine, Technical University Munich, München, Germany, ${ }^{4}$ Department of Cardiology, Municipal Hospital Triemli, Zurich, Switzerland, ${ }^{5}$ Department of Neurology, Azienda Unità Sanitaria Locale-IRCCS, Reggio Emilia, Italy, ${ }^{6}$ Department of Neurology, University Hospitals of Leuven, Leuven, Belgium, ${ }^{7}$ Stroke Division, Florey Institute of Neuroscience and Mental Health, University of Melbourne, Melbourne, VIC, Australia, ${ }^{8}$ Department of Neurology, Alfried-Krupp Krankenhaus, Essen, Germany, ${ }^{9}$ Department of Neurology, Heinrich Heine University Duesseldorf, Duesseldorf, Germany, ${ }^{10}$ Department of Neurology, University Hospital of Basel, Basel, Switzerland, " 11 Felix-Platter Hospital, Basel, Switzerland, ${ }^{12}$ Department of Neurology, University Hospital of Girona, Girona, Spain, ${ }^{13}$ Department of Neurology, University Hospital of Essen, Essen, Germany, ${ }^{14}$ Department of Neurology, Klinikum Worms, Worms, Germany, ${ }^{15}$ Department of Neurology, University Hospital of Zurich, Zurich, Switzerland, ${ }^{16}$ Department of Neurology, University Hospital of Ghent, Ghent, Belgium, ${ }^{17}$ Department of Neurology, Tufts Medical Center, Boston, MA, United States, ${ }^{18}$ Ammerland Klinik, Westerstede, Germany, ${ }^{19}$ Department of Neurology, AZ Sint Blasius, Dendermonde, Belgium, ${ }^{20}$ Department of Neurology, University Hospital of Geneva, Geneva, Switzerland, ${ }^{21}$ Department of Neurology, Baystate Health Center, Springfield, MA, United States, ${ }^{22}$ Sackler School of Medicine and Department of Neurology, Yitzchak Shamir Medical Center, Tel Aviv University, Tel Aviv-Yafo, Israel, ${ }^{23}$ Institute for Clinical Research and Health Policy Studies, Tufts Medical Center, Boston, MA, United States, ${ }^{24}$ Department of Cardiology, University Hospital of Bern, Bern, Switzerland, ${ }^{25}$ Department of Neurology, University Hospital of Bern, Bern, Switzerland

Background and Purpose: To identify factors associated with prior stroke at presentation in patients with cryptogenic stroke (CS) and patent foramen ovale (PFO).

Methods: We studied cross-sectional data from the International PFO Consortium Study (NCT00859885). Patients with first-ever stroke and those with prior stroke at baseline were analyzed for an association with PFO-related (right-to-left shunt at rest, atrial septal aneurysm, deep venous thrombosis, pulmonary embolism, and Valsalva maneuver) and PFO-unrelated factors (age, gender, BMI, hypertension, diabetes mellitus, hypercholesterolemia, smoking, migraine, coronary artery disease, aortic plaque). A multivariable analysis was used to adjust effect estimation for confounding, e.g., owing to the age-dependent definition of study groups in this cross-sectional study design.

Results: We identified 635 patients with first-ever and 53 patients with prior stroke. Age, BMI, hypertension, diabetes mellitus, hypercholesterolemia, coronary artery disease, and right-to-left shunt $(R L S)$ at rest were significantly associated with prior stroke. Using a pre-specified multivariable logistic regression model, age (Odds Ratio 1.06), BMI (OR 1.06), hypercholesterolemia (OR 1.90) and RLS at rest (OR 1.88) were strongly associated with prior stroke.Based on these factors, we developed a nomogram to illustrate the strength of the relation of individual factors to prior stroke. 
Conclusion: In patients with CS and PFO, the likelihood of prior stroke is associated with both, PFO-related and PFO-unrelated factors.

Keywords: patent foramen ovale, PFO, right-to-left shunt, cryptogenic stroke, prior stroke, risk factor, hypercholesterolemia, International PFO Consortium

\section{INTRODUCTION}

The prevalence of patent foramen ovale (PFO) in the general adult population is $15-35 \%$ (1) and its association with cryptogenic stroke (CS) has been clearly established $(2,3)$. The higher prevalence of PFO in CS of all ages $(3,4)$ suggests a pathogenic role for $\mathrm{PFO}$, at least in a substantial portion of these patients. Assuming that paradoxical embolism is the predominant pathogenic mechanism for recurrent strokes (5), PFO closure is a logical treatment option. However, recent RCTs comparing percutaneous closure with antithrombotic treatment revealed inconsistent results-some of them in favor of closure (6-9), whereas others without a significant advantage of closure (10-13). Low recurrence rates under both prevention regimens, non-PFO related recurrent stroke mechanisms, crossovers, procedure- and device-related complications as well as suboptimal patient selection-i.e., including some patients with non-PFO-related index strokes-might explain the inconsistency of the results (14-17). Hence, in patients with PFO and CS, the risk of stroke recurrence may be associated with both PFO-related and PFO-unrelated factors.

Previous strokes at presentation have been identified as a risk factor for stroke recurrence in patients with CS and PFO (18).

The aim of this study was to identify PFO-related and unrelated risk factors associated with prior stroke in CS patients. Furthermore, we developed a nomogram to illustrate the strength of these associations.

\section{METHODS}

\section{Patients}

The International PFO Consortium is an ongoing academic trial, where researchers from currently nineteen stroke centers worldwide collaborate (NCT00859885). It was founded in 2008 and collects data of patients with ischemic stroke or TIA and PFO. Emphasis is placed on the evaluation of risk factors, PFO diagnosis, and secondary stroke prevention. It is a multicenter prospective study with a scheduled yearly follow-up. Database is expected to be closed after all patients reach a minimum of three years follow-up in 2021. Ethical approval was obtained from the local ethics committee of the corresponding center if legally required.

Patients older than 18 years with ischemic stroke or TIA $\leq 3$ months and proven PFO on transesophageal echocardiography are eligible for the International PFO Consortium Study. There was no upper age limit. The whole International PFO consortium cohort included patients with different stroke etiologies. In the current study we addressed those with an undetermined stroke etiology, i.e., CS. Baseline data comprise demographic data, vascular risk factors, conditions predisposing to paradoxical embolism, previous medication, brain CT or MRI findings, echocardiographic PFO-features, and stroke etiology according to TOAST criteria (19). Annual follow-up visits assess secondary stroke prevention and stroke recurrence. Vascular risk factors include age, gender, arterial hypertension, diabetes mellitus, hypercholesterolemia, smoking, self-reported migraine, coronary artery disease, previous stroke, thrombophilia (factor V Leiden and prothrombin mutation, protein $\mathrm{C}$ and $\mathrm{S}$ deficiency, AT3 deficiency, and antiphospholipid antibodies). Echocardiographic features include atrial septal aneurysm (ASA) defined as hypermobility of the atrial septum with an excursion of $\geq 10 \mathrm{~mm}$ from midline, aortic plaques $>4 \mathrm{~mm}$ thickness, and right-toleft shunt (RLS) at rest or under Valsalva maneuver (VM). Conditions predisposing to paradoxical embolism comprise VM at the time of stroke onset, deep vein thrombosis (DVT), and pulmonary embolism.

From September 2008 through December 2014, the International PFO Consortium enrolled 931 patients with CS and PFO. The present study focused on two patient subgroups: (a) 635 patients with first-ever stroke (i.e., neither radiological nor clinical evidence of prior stroke) and (b) 53 patients with prior stroke (i.e., both clinical and radiological evidence of prior stroke). Patients, who could not unambiguously assigned to the first-ever or the recurrent stroke group on the basis of past medical history and radiological signs, i.e., CS patients with clinical but no radiological evidence of prior stroke or vice versa $(n=243)$ were not included in the present study.

\section{Statistical Analysis}

The distribution of quantitative data is described by mean \pm standard deviation. Qualitative data is presented by absolute and relative frequencies. Corresponding hypothesis testing was performed by $t$-Test and the Chi-squared test or Fisher's exact test, as appropriate.

Missing values were imputed using a Random Forests model to account for possible interactions and high-dimensional relations of the data (20). Associations with prior stroke were estimated by Odds Ratios, with 95\% confidence intervals, using univariate and multivariable logistic regression models. Any model contained age as an independent variable to adjust for confounding by the time-dependent stroke risk. Therefore, each estimated effect is conditioned on age, i.e., the assessment of PFOrelated and-unrelated factors is valid for patients of the same age who are consequently at the same time-dependent stroke risk. The multivariable model was pre-specified to avoid bias and an increased risk of data-driven false-positive findings (21).

A nomogram was developed to illustrate the effect size of factors. Hypothesis testing was performed on exploratory twosided 5\% significance levels. 
Of note, our main research goal was identification and effect estimation of potential risk factors rather than hypothesis testing. Moreover, the current study design did not allow for sample size calculation and thus might not have been adequately powered to test the multiple null hypotheses that the respective regression coefficients are zero.

All analyses were performed using the statistical software $\mathrm{R}$ 3.6.1 (The $\mathrm{R}$ Foundation for Statistical Computing, Vienna, Austria).

\section{RESULTS}

\section{Factors Associated With Prior Stroke}

Patient baseline characteristics are shown in Table 1. CS patients with prior stroke were significantly older $(64.8 \pm 10.8$ vs. 53.3 \pm 14 years), showed a higher body-mass-index (BMI, $27.8 \pm$ 4.9 vs. $25.7 \pm 4.5$ ), were more likely to suffer from hypertension ( 59 vs. $32 \%$ ), diabetes mellitus (19 vs. 6\%), hypercholesterolemia (72 vs. $49 \%$ ), and coronary artery disease (11 vs. $5 \%$ ) and had a higher portion of right-to-left shunt (RLS) at rest (43 vs. 28\%) compared to those with first-ever stroke. Adjusting for age, the odds ratio for these factors in the univariable model was 1.07, $1.09,2.93,3.37,2.67,2.57$, and 2.00 for RLS at rest, respectively (Table 2). As expected, patients with prior stroke were frequently on antithombotic (72 vs. first-ever stroke 12\%), antihypertensive (51 vs. $23 \%$ ) and lipid lowering drugs ( 49 vs. $10 \%$; all $p<0.0001$ ).

The pre-specified multivariable logistic regression (Table 3 ) demonstrated that prior stroke was strongly associated with advancing age (OR 1.06, 95\%CI 1.04-1.10, $p<0.001$ ), RLS at rest (OR 1.88, 95\%CI 1.00-3.47, $p=0.046)$, hypercholesterolemia (OR 1.90, 95\%CI 1.00-3.73, $p=0.055)$ and BMI (OR 1.06, 95\%CI $0.99-1.13, p=0.074)$, reaching statistical significance for age

TABLE 2 | Association of baseline characteristics with prior stroke-univariate analysis (missing values were imputed).

\begin{tabular}{llll}
\hline Predictor variable & OR & $\mathbf{9 5 \% ~ C l}$ & $\boldsymbol{P}$-value \\
\hline Age, years & 1.07 & $1.05-1.10$ & $<0.001$ \\
Male gender & 0.86 & $0.48-1.52$ & 0.617 \\
Body-mass-index & 1.09 & $1.03-1.14$ & 0.002 \\
Hypertension & 2.93 & $1.67-5.25$ & $<0.001$ \\
Diabetes mellitus & 3.37 & $1.51-6.96$ & 0.002 \\
Hypercholesterolemia & 2.67 & $1.47-5.10$ & 0.002 \\
Smoking & 0.73 & $0.35-1.40$ & 0.365 \\
Migraine & 0.80 & $0.38-1.53$ & 0.519 \\
Coronary artery disease & 2.57 & $0.93-6.11$ & 0.045 \\
Aortic plaque & 2.32 & $0.53-7.26$ & 0.192 \\
Valsalva maneuver & 0.22 & $0.01-1.04$ & 0.138 \\
Deep vein thrombosis & 1.77 & $0.51-4.74$ & 0.304 \\
Pulmonary embolism & 0.85 & $0.05-4.37$ & 0.879 \\
Right-to-left shunt at rest & 2.00 & $1.12-3.53$ & 0.017 \\
Atrial septal aneurysm & 1.18 & $0.65-2.10$ & 0.568 \\
\hline
\end{tabular}

OR, odds ratio; $\mathrm{Cl}$, confidence interval.

TABLE 1 | Baseline demographic, clinical and imaging data (missing values were imputed).

\begin{tabular}{|c|c|c|c|c|c|}
\hline & First-ever stroke $n=635$ & Missing values & Prior stroke $n=53$ & Missing values & $P$-value \\
\hline Age, years & $53.3 \pm 14.0$ & - & $64.8 \pm 10.8$ & - & $<0.001$ \\
\hline Male gender & $262(41.3)$ & - & $20(37.7)$ & - & 0.722 \\
\hline Body mass index & $25.7 \pm 4.5$ & 29 & $27.8 \pm 4.9$ & 2 & 0.003 \\
\hline Hypertension & $206(32.4)$ & 1 & $31(58.5)$ & - & $<0.001$ \\
\hline Diabetes mellitus & $41(6.5)$ & 2 & $10(18.9)$ & - & 0.003 \\
\hline Migraine & $157(24.7)$ & 34 & $11(20.8)$ & 4 & 0.631 \\
\hline Coronary artery disease & $30(4.7)$ & 8 & $6(11.3)$ & 1 & 0.050 \\
\hline Aortic plaque & $16(2.5)$ & - & $3(5.7)$ & - & 0.174 \\
\hline Valsalva maneuver & $51(8.0)$ & 57 & $1(1.9)$ & 4 & 0.169 \\
\hline Deep vein thrombosis & $27(4.5)$ & 31 & $4(7.7)$ & 1 & 0.298 \\
\hline \multicolumn{6}{|l|}{ Medication on admission } \\
\hline Antithrombotic therapy & $77(11.9)$ & & $38(71.7)$ & & $<0.001$ \\
\hline Antiplatelet & 66 & & 35 & & \\
\hline Oral anticoagulation & 11 & & 3 & & \\
\hline Antihypertensive drugs & $145(22.5)$ & & 27 (50.9) & & $<0.001$ \\
\hline Lipid lowering drugs & 65 (10.1) & & $26(49.1)$ & & $<0.001$ \\
\hline
\end{tabular}

Values are mean $\pm S D$ or $n(\%)$. 
TABLE 3 | Association of baseline characteristics with prior stroke-multivariable analysis (pre-specified, missing values were imputed).

\begin{tabular}{lccc}
\hline Predictor variable & OR & $\mathbf{9 5 \%} \mathbf{C l}$ & $\boldsymbol{P}$-value \\
\hline Age, years & 1.06 & $1.04-1.10$ & $<0.001$ \\
Male gender & 0.78 & $0.41-1.46$ & 0.446 \\
Body-mass-index & 1.06 & $0.99-1.13$ & 0.074 \\
Hypertension & 1.06 & $0.53-2.11$ & 0.872 \\
Diabetes mellitus & 1.45 & $0.58-3.42$ & 0.413 \\
Hypercholesterolemia & 1.90 & $1.00-3.73$ & 0.055 \\
Smoking & 1.35 & $0.61-2.82$ & 0.439 \\
Valsalva maneuver & 0.28 & $0.02-1.39$ & 0.218 \\
Deep vein thrombosis & 1.76 & $0.46-5.44$ & 0.361 \\
Right-to-left shunt at rest & 1.88 & $1.00-3.47$ & 0.046 \\
Atrial septal aneurysm & 0.98 & $0.51-1.84$ & 0.959 \\
\hline
\end{tabular}

OR, odds ratio; $\mathrm{Cl}$, confidence interval.

and RLS at rest. Moreover, the presence of a DVT (OR 1.76, $95 \%$ CI $0.46-5.44, p=0.361)$ as well as an absent VM just before stroke onset (OR $0.28,95 \%$ CI $0.02-1.39, p=0.218$ ) also hinted at a strong association with prior stroke, but was not statistically significant in this cross-sectional analysis.

Considering the weight of each predictor variable in the pre-specified multivariable model, reflected by its Odds Ratio, we developed a nomogram to illustrate the strength of each relation to prior stroke (Figure 1). Accordingly, age, BMI, hypercholesterolemia, RLS at rest, absence of VM directly preceding stroke onset and the presence of a DVT are the main factors associated with stroke recurrence.

For example, a 55-year-old (+40points) female (+10p) CS patient with $\mathrm{PFO}$ and an RLS at rest $(+27.5 \mathrm{p})$, BMI 30 $\mathrm{kg} / \mathrm{m}^{2}(+50 \mathrm{p})$, presence of VM just before stroke onset (0p), sonographic proof of ASA (0p) and DVT $(+25 \mathrm{p})$, known hypercholesterolemia $(+27.5 p)$, no arterial hypertension $(0 p)$ or diabetes (0p) and non-smoker (0p) sums up to a total of 180 points, which corresponds to a likelihood of $7-8 \%$ that this women belongs to the patient group with prior stroke.

\section{DISCUSSION}

The present analysis revealed associations of prior stroke with both PFO-related and -unrelated risk factors. Our study gives a novel insight into the nature and strength of the relationship of previous strokes at presentation and PFO.

Previous clinical and/or radiological stroke at presentation has been associated with higher risk of stroke recurrence in some studies (18) but not in others (22). In addition, recent data suggest that only CS patients with PFO in the high Risk of Paradoxical Embolism (RoPE)- Score strata, i.e., absence of classical vascular risk factors such as hypertension, diabetes mellitus and advancing age show an association of prior stroke with stroke recurrence (15). Age might play a dual role in the pathogenesis of stroke recurrence-both as a $\mathrm{PFO}$-unrelated and $\mathrm{PFO}$-related factor. It is usually considered a stroke risk factor that operates through PFO-unrelated pathogenic mechanisms. The increasing prevalence of classical vascular risk factors in older patients and the fact that stroke recurrence after PFO closure was higher in patients $>55$ years of age than in younger patients underlines the relevance of PFO-unrelated contributors to stroke recurrence $(23,24)$. On the other hand, age might also increase the PFOrelated stroke risk by prolonging the exposure time to Right-toLeft-Shunt. Prothrombotic conditions like endothelial damage, hypercoagulability, chronic inflammation, and venous stasis due to decreased regular exercise, which may not be addressed during routine stroke workup or may even be undetectable, accumulate with age and can predispose to paradoxical embolism in the long term (25).

The association of PFO-related factors with stroke recurrence has never been reliably established. Large PFOs have been positively associated with stroke recurrence in some studies (2628) but not in others $(14,15,29-31)$. The recent CLOSE and DEFENSE trials $(7,9)$ enrolled carefully selected cryptogenic stroke patients with large PFOs or concomitant atrial septal aneurysm. The studies showed that PFO closure was more efficacious in reducing the risk of stroke recurrence than antithrombotic treatment alone. The GORE-REDUCE trial included predominantly patients with moderate to large RLS and likewise demonstrated the superiority of PFO closure over medical treatment alone in preventing recurrent stroke (8). In addition, recent meta-analyses of RCTs comparing percutaneous PFO device closure with medical therapy in CS patients further support device closure in patients with certain PFO characteristics in particular moderate to large shunts $(32,33)$. Since PFO closure cannot prevent strokes of other possible etiologies, the findings of the above studies further emphasize the role of PFO-related factors in the pathogenesis of stroke recurrence.

Although our data suggest a strong association of prior stroke with conditions predisposing to paradoxical embolism such as DVT (OR 1.76) and VM directly preceding stroke onset (OR 0.28 ), the evidence is currently weak (DVT $p=0.361, \mathrm{VM}$ $p=0.218$ ) and needs confirmation in prospective, adequately powered trials. Briefly, the prevalence of DVT in the lower extremities, which was systematically captured in our database, was $4.4 \%$ in patients with first-ever stroke and $7.6 \%$ in patients with prior stroke. The findings are in keeping with the results of previous studies (34). However, we did not assess the prevalence of pelvic vein thrombosis in all patients. Paradoxical emboli originating from the pelvis have been recognized as an alternate source of stroke in this population (35). The missing data on pelvic vein thrombosis as well as the cross sectional study design may have obfuscated a statistical significant association between DVT and prior stroke.

VM at stroke onset was associated with a $72 \%$ reduced likelihood of a previous ischemic event. This could be best explained by the fact that VM increases RLS volume and supports a causal relationship between stroke and PFO, i.e. the stroke is most likely attributable to the PFO. PFO attributable strokes in turn demonstrated a low recurrence rate (36).

In terms of PFO-unrelated factors, our study identified hypercholesterolemia (OR 1.90, $p=0.055$ ) and higher BMI 


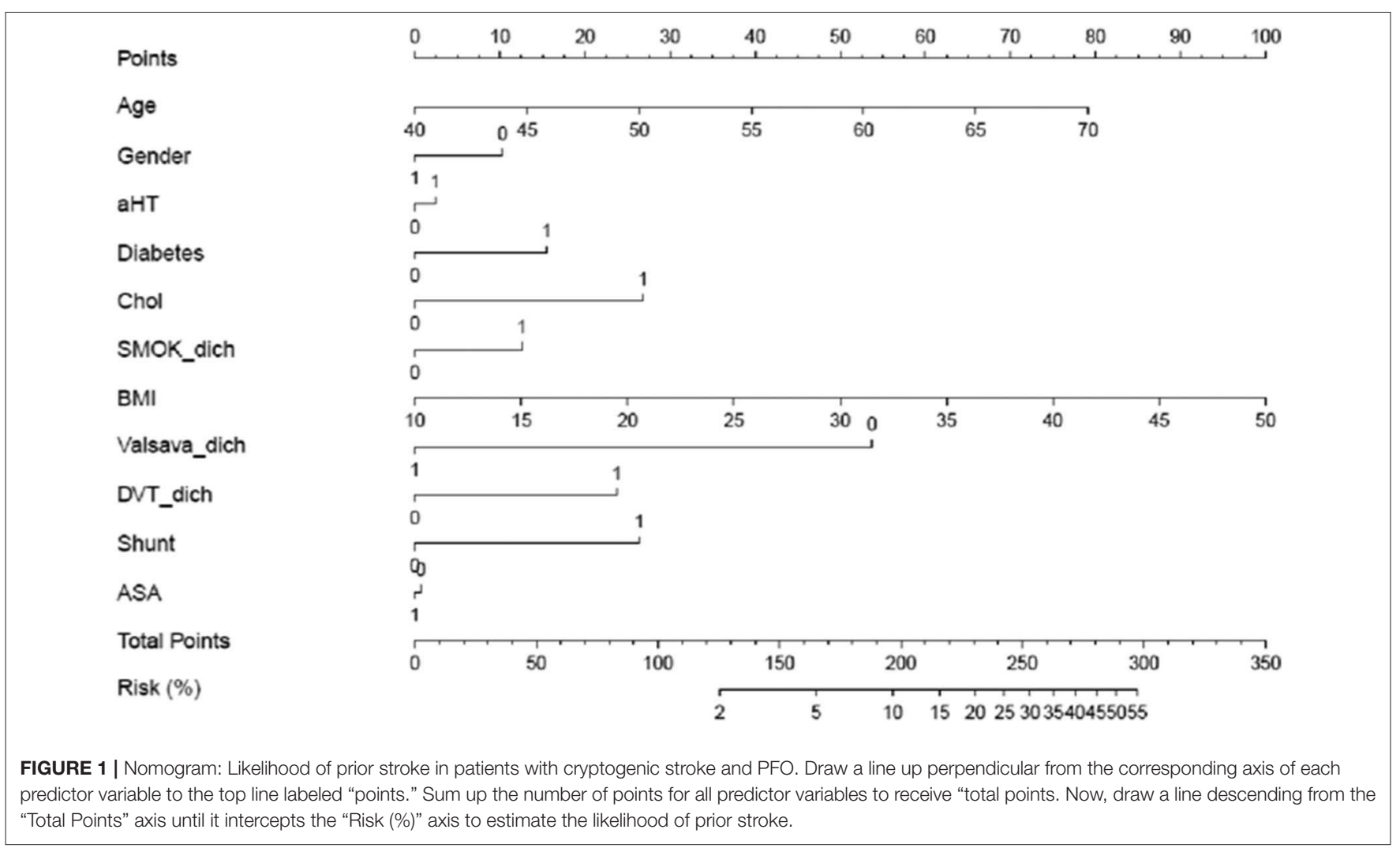

(OR 1.06, $p=0.074$ ) as being strongly associated with prior stroke, albeit not adequately powered to demonstrate statistical significance. Hyperlipidemia, especially an elevated ratio of ApoE/A1 or non-HDL/HDL levels, are known risk factors for ischemic stroke (37). Lipid-lowering drugs are firmly established in secondary stroke prevention (38). Just recently, it was shown that lowering LDL-levels below $1.8 \mathrm{mmol} / \mathrm{l}$ after stroke/TIA reduces the risk of a subsequent cardiovascular event compared to higher target LDL-levels (39), and the new ESCguidelines recommend even lower LDL-levels in selected highrisk patients (40).

Several observational studies point to a lower rate of stroke recurrence in overweight or obese patients (41-44). However, recent studies in stroke patients receiving intravenous thrombolysis or patients with mild symptoms did not detect this relationship, thus challenging the "obesity paradox" (45, 46). Obesity was more common among patients with multiple $\mathrm{CS}$ and $\mathrm{PFO}$ in a single study, though the recurrence risk was not independently associated with BMI (18). Given these controversial findings, the impact of BMI on stroke recurrence needs further elucidation. Particularly in CS patients with PFO, elevated BMI and the presence of obstructive sleep apnea (OSA) might play a relevant role. Just recently, the coexistence of PFO and OSA in overweight men was suggested as a risk factor for wake-up stroke (47). Moreover, prolonged OSA episodes promoted RLS occurrence during sleep, which might increase the exposure time for paradoxical embolism (48).
The present study is limited by the missing assessment of OSA and other potentially high-risk PFO characteristics such as the presence of an Eustachian valve, a Chiari network or left atrial enlargement (49). In addition, the International PFO consortium study did not collect data on history of migraine stratified into those with aura or without. Furthermore, in patients with prior stroke, the PFO features were assessed at the time of the recurrent stroke only (i.e., at study enrollment). However, it is very unlikely that shunt size or presence of ASA would have changed substantially over time. Third, the process of screening for PFO across the 19 participating stroke centers was not standardized and thus might differ. Fourth, the effect of age cannot be separated from the time-dependent stroke risk. Therefore, age was mainly considered a confounder to allow adjusted effect estimation of other risk factors considered in the models.

Finally, we developed a nomogram to better illustrate the effect size of each risk factor and to easily estimate the probability of having suffered from a prior ischemic event at the time of the index stroke. Several studies suggest that prior stroke might also be associated with stroke recurrence $(50,51)$. Due to the cross sectional design of our study, we are currently not able to firmly establish those factors as risk factors for future events. However, the present study allows to identify promising "risk factor" candidates for recurrent stroke to be then tested in a longitudinal study design. 


\section{CONCLUSION}

In CS patients with PFO, RLS at rest, hypercholesterolemia and higher BMI were strongly related to prior stroke. The likelihood of prior stroke is associated with both, PFO-related and -unrelated factors. Based on the present findings, the impact of these factors on stroke recurrence in CS patients with PFO need to be further established in a longitudinal study design now.

\section{DATA AVAILABILITY STATEMENT}

Data that is not available with the article will be provided in an anonymized form by the corresponding author upon reasonable request from any qualified investigator (subject to the provisions of the IRB).

\section{ETHICS STATEMENT}

The studies involving human participants were reviewed and approved by Ethikkommission Nordwest- und Zentralschweiz (EKNZ 2014-031). The patients/participants

\section{REFERENCES}

1. Homma S, Messé SR, Rundek T, Sun Y-P, Franke J, Davidson K, et al. Patent foramen ovale. Nat Rev Dis Primers. (2016) 2:15086. doi: $10.1038 / \mathrm{nrdp} .2015 .86$

2. Pristipino C, Sievert H, D’Ascenzo F, Mas JL, Meier B, Scacciatella P, et al. European position paper on the management of patients with patent foramen ovale. General approach and left circulation thromboembolism. Euro Interv. (2019) 14:1389-402. doi: 10.4244/EIJ-D-18-00622

3. Mazzucco S, Li L, Binney L, Rothwell PM. Prevalence of patent foramen ovale in cryptogenic transient ischaemic attack and non-disabling stroke at older ages: a population-based study, systematic review, and meta-analysis. Lancet Neurol. (2018) 17:609-617. doi: 10.1016/S1474-4422(18)30167-4

4. Mattle HP, Saver JL. Patent foramen ovale increases stroke risk in older people. Nat Rev Neurol. (2018) 14:573-4. doi: 10.1038/s41582-018-0050-7

5. Shang X, Li D, Qiu Q, Xiao S, Wang L, Zhang C, et al. First direct evidence of a Patent Foramen Ovale (PFO): a large thrombus straddling the foramen ovale. Eur Heart J. (2016) 37:782. doi: 10.1093/eurheartj/ehv380

6. Saver JL, Carroll JD, Thaler DE, Smalling RW, MacDonald LA, Marks DS, et al. Long-term outcomes of patent foramen ovale closure or medical therapy after stroke. N Engl J Med. (2017) 377:1022-32. doi: 10.1056/NEJMoa1610057

7. Mas J-L, Derumeaux G, Guillon B, Massardier E, Hosseini H, Mechtouff L, et al. Patent foramen ovale closure or anticoagulation vs. antiplatelets after stroke. N Engl J Med. (2017) 377:1011-21. doi: 10.1056/NEJMoa1705915

8. Søndergaard L, Kasner SE, Rhodes JF, Andersen G, Iversen HK, Nielsen-Kudsk JE, et al. Patent foramen ovale closure or antiplatelet therapy for cryptogenic stroke. N Engl J Med. (2017) 377:1033-42. doi: 10.1056/NEJMoa1707404

9. Lee PH, Song J-K, Kim JS, Heo R, Lee S, Kim D-H, et al. Cryptogenic stroke and high-risk patent foramen ovale: The DEFENSE-PFO trial. J Am Coll Cardiol. (2018) 71:2335-42. doi: 10.1016/j.jacc.2018.02.046

10. Carroll JD, Saver JL, Thaler DE, Smalling RW, Berry S, MacDonald LA, et al. Closure of patent foramen ovale versus medical therapy after cryptogenic stroke. N Engl J Med. (2013) 368:1092-100. doi: 10.1056/NEJMoa1301440

11. Meier B, Kalesan B, Mattle HP, Khattab AA, Hildick-Smith D, Dudek D, et al. Percutaneous closure of patent foramen ovale in cryptogenic embolism. $N$ Engl J Med. (2013) 368:1083-91. doi: 10.1056/NEJMoa1211716 provided their written informed consent to participate in this study.

\section{AUTHOR CONTRIBUTIONS}

TK, M-LM, and KN contributed conception and design of the study. AH performed the final statistical analysis. TK and $\mathrm{KN}$ drafted the manuscript and all authors critically revised it for important intellectual content. All authors substantially contributed to the acquisition, analysis or interpretation of data and approved the final version of the manuscript.

\section{FUNDING}

This work was supported by the Swiss National Science Foundation [grant number 32003B_120522], Swiss Heart Foundation, Cantonal Hospital Aarau Research Foundation [grant number 1410.000.049].

\section{ACKNOWLEDGMENTS}

We would like to thank Sandra Clarke, Barbara Mangold and Sylvie Nuc for administrative assistance.

12. Furlan AJ, Reisman M, Massaro J, Mauri L, Adams H, Albers GW, et al. Closure or medical therapy for cryptogenic stroke with patent foramen ovale. N Engl J Med. (2012) 366:991-9. doi: 10.1056/NEJMoa1009639

13. Ntaios G, Papavasileiou V, Makaritsis K, Michel P. PFO closure vs. medical therapy in cryptogenic stroke or transient ischemic attack: a systematic review and meta-analysis. Int J Cardiol. (2013) 169:101-5. doi: 10.1016/j.ijcard.2013.08.058

14. Mono M-L, Geister L, Galimanis A, Jung S, Praz F, Arnold M, et al. Patent foramen ovale may be causal for the first stroke but unrelated to subsequent ischemic events. Stroke. (2011) 42:2891-5. doi: 10.1161/STROKEAHA.111.619577

15. Thaler DE, Ruthazer R, Weimar C, Mas J-L, Serena J, Di Angelantonio E, et al. Recurrent stroke predictors differ in medically treated patients with pathogenic vs. other PFOs. Neurology. (2014) 83:221-6. doi: 10.1212/WNL.0000000000000589

16. Bang OY, Lee MJ, Ryoo S, Kim SJ, Kim JW. Patent foramen ovale and strokecurrent status. J Stroke. (2015) 17:229-37. doi: 10.5853/jos.2015.17.3.229

17. Köhrmann M, Schellinger PD, Tsivgoulis G, Steiner T. Patent foramen ovale: story closed? J Stroke. (2019) 21:23-30. doi: 10.5853/jos.2018.03097

18. Nedeltchev K. Outcome of patients with cryptogenic stroke and patent foramen ovale. J Neurol Neurosurg Psychiatry. (2002) 72:347-50. doi: 10.1136/jnnp.72.3.347

19. Adams HP, Bendixen BH, Kappelle LJ, Biller J, Love BB, Gordon DL, et al. Classification of subtype of acute ischemic stroke. Definitions for use in a multicenter clinical trial. TOAST. Trial of Org 10172 in Acute Stroke Treatment. Stroke. (1993) 24:35-41. doi: 10.1161/01.STR.2 4.1 .35

20. Stekhoven DJ, Bühlmann P. MissForest-non-parametric missing value imputation for mixed-type data. Bioinformatics. (2012) 28:112-8. doi: 10.1093/bioinformatics/btr597

21. Vittinghoff E, McCulloch CE. Relaxing the rule of ten events per variable in logistic and Cox regression. Am J Epidemiol. (2007) 165:710-8. doi: 10.1093/aje/kwk052

22. Almekhlafi MA, Wilton SB, Rabi DM, Ghali WA, Lorenzetti DL, Hill MD. Recurrent cerebral ischemia in medically treated patent foramen ovale: a meta-analysis. Neurology. (2009) 73:89-97. doi: 10.1212/WNL.0b013e3181aa2a19 
23. Luermans JG, Budts W, Ten Berg JM, Plokker HW, Suttorp MJ, Post MC. Comparison of outcome after patent foramen ovale closure in older versus younger patients. EuroIntervention. (2011) 7:209-15. doi: 10.4244/EIJV7I2A35

24. Scacciatella P, Meynet I, Presbitero P, Giorgi M, Lucarelli C, Zavalloni Parenti $\mathrm{D}$, et al. Recurrent cerebral ischemia after patent foramen ovale percutaneous closure in older patients: A two-center registry study. Catheter Cardiovasc Interv. (2016) 87:508-14. doi: 10.1002/ccd.26053

25. Rumley A, Emberson JR, Wannamethee SG, Lennon L, Whincup PH, Lowe GDO. Effects of older age on fibrin D-dimer, C-reactive protein, and other hemostatic and inflammatory variables in men aged 60-79 years. J Thromb Haemost. (2006) 4:982-7. doi: 10.1111/j.1538-7836.2006.01889.x

26. Anzola GP, Zavarize P, Morandi E, Rozzini L, Parrinello G. Transcranial Doppler and risk of recurrence in patients with stroke and patent foramen ovale. Eur J Neurol. (2003) 10:129-35. doi: 10.1046/j.1468-1331.2003.00561.x

27. Fukuoka T, Dembo T, Nagoya H, Kato Y, Yasuko O, Deguchi I, et al. Factors related to recurrence of paradoxical cerebral embolism due to patent foramen ovale. J Neurol. (2012) 259:1051-5. doi: 10.1007/s00415-011-6297-1

28. Rigatelli G, Magro B, Oliva L. Anatomo-functional characterization of interatrial septum for catheter-based interventions. Am J Cardiovasc Dis. (2011) 1:227-35.

29. Homma S, Sacco RL, Di Tullio MR, Sciacca RR, Mohr JP, PFO in Cryptogenic Stroke Study (PICSS) Investigators. Effect of medical treatment in stroke patients with patent foramen ovale: patent foramen ovale in Cryptogenic Stroke Study. Circulation. (2002) 105:2625-631. doi: 10.1161/01.CIR.0000017498.88393.44

30. Serena J, Marti-Fàbregas J, Santamarina E, Rodríguez JJ, Perez-Ayuso MJ, Masjuan J, et al. Recurrent stroke and massive right-to-left shunt: results from the prospective Spanish multicenter (CODICIA) study. Stroke. (2008) 39:3131-6. doi: 10.1161/STROKEAHA.108.521427

31. Katsanos AH, Spence JD, Bogiatzi C, Parissis J, Giannopoulos S, Frogoudaki A, et al. Recurrent stroke and patent foramen ovale: a systematic review and meta-analysis. Stroke. (2014) 45:3352-9. doi: 10.1161/STROKEAHA.114.007109

32. Reinthaler M, Ozga A-K, Sinning D, Curio J, Al-Hindwan HS, Bäckemo Johansson J, et al. Revival of transcatheter PFO closure: A meta-analysis of randomized controlled trials - impact of shunt size and age. Am Heart J. (2018) 201:95-102. doi: 10.1016/j.ahj.2018.03.025

33. Ahmad Y, Howard JP, Arnold A, Shin MS, Cook C, Petraco R, et al. Patent foramen ovale closure vs. medical therapy for cryptogenic stroke: a metaanalysis of randomized controlled trials. Eur Heart J. (2018) 39:1638-49. doi: 10.1093/eurheartj/ehy121

34. Lapergue B, Decroix JP, Evrard S, Wang A, Bendetowicz D, Offroy MA, et al. Diagnostic yield of venous thrombosis and pulmonary embolism by combined CT venography and pulmonary angiography in patients with cryptogenic stroke and patent foramen ovale. Eur Neurol. (2015) 74:69-72. doi: $10.1159 / 000437261$

35. Osgood M, Budman E, Carandang R, Goddeau RP Jr, Henninger N. Prevalence of pelvic vein pathology in patients with cryptogenic stroke and patent foramen ovale undergoing MRV pelvis. Cerebrovasc Dis. (2015) 39:216-23. doi: 10.1159/000376613

36. Kent DM, Ruthazer R, Weimar C, Mas J-L, Serena J, Homma S, et al. An index to identify stroke-related vs incidental patent foramen ovale in cryptogenic stroke. Neurology. (2013) 81:619-25. doi: 10.1212/WNL.0b013e3182a08d59

37. O’Donnell MJ, Xavier D, Liu L, Zhang H, Chin SL, Rao-Melacini P, et al. Risk factors for ischaemic and intracerebral haemorrhagic stroke in 22 countries (the INTERSTROKE study): a case-control study. Lancet. (2010) 376:112-23. doi: 10.1016/S0140-6736(10)60834-3

38. Hankey GJ. Secondary stroke prevention. Lancet Neurol. (2014) 13:178-94. doi: 10.1016/S1474-4422(13)70255-2

39. Amarenco P, Kim JS, Labreuche J, Charles H, Abtan J, Béjot Y, et al. A Comparison of two LDL cholesterol targets after ischemic stroke. $N$ Engl J Med. (2020) 382:9. doi: 10.1056/NEJMoa1910355
40. Mach F, Baigent C, Catapano AL, Koskinas KC, Casula M, Badimon L, et al. 2019 ESC/EAS Guidelines for the management of dyslipidaemias: lipid modification to reduce cardiovascular risk. Eur Heart J. (2020) 41:111-88. doi: 10.1093/eurheartj/ehz455

41. Doehner W, Schenkel J, Anker SD, Springer J, Audebert HJ. Overweight and obesity are associated with improved survival, functional outcome, and stroke recurrence after acute stroke or transient ischaemic attack: observations from the TEMPiS trial. Eur Heart J. (2013) 34:268-77. doi: 10.1093/eurheartj/ehs340

42. Oesch L, Tatlisumak T, Arnold M, Sarikaya H. Obesity paradox in stroke - Myth or reality? A systematic review. PLoS ONE. (2017) 12:e0171334. doi: 10.1371/journal.pone.0171334

43. Huang K, Liu F, Han X, Huang C, Huang J, Gu D, et al. Association of BMI with total mortality and recurrent stroke among stroke patients: A meta-analysis of cohort studies. Atherosclerosis. (2016) 253:94-101. doi: 10.1016/j.atherosclerosis.2016.08.042

44. Andersen KK, Olsen TS. The obesity paradox in stroke: lower mortality and lower risk of readmission for recurrent stroke in obese stroke patients. Int J Stroke. (2015) 10:99-104. doi: 10.1111/ijs.12016

45. Branscheidt M, Schneider J, Michel P, Eskioglou E, Kaegi G, Stark R, et al. No impact of body mass index on outcome in stroke patients treated with iv thrombolysis BMI and IV thrombolysis outcome. PLoS ONE. (2016) 11:e0164413. doi: 10.1371/journal.pone.0164413

46. Chen W, Pan Y, Jing J, Zhao X, Liu L, Meng X, et al. Association of body mass index and risk of stroke after acute minor stroke or tia: a post hoc analysis of a randomized controlled trial. Neurotox Res. (2019) 36:836-43. doi: 10.1007/s12640-019-00056-4

47. Man H, Xu Y, Zhao Z, Zhang S, Lv R, Chi X, et al. The coexistence of a patent foramen ovale and obstructive sleep apnea may increase the risk of wake-up stroke in young adults. Technol Health Care. (2019) 27:23-30. doi: 10.3233/THC-199004

48. Beelke M, Angeli S, Del Sette M, De Carli F, Canovaro P, Nobili L, et al. Obstructive sleep apnea can be provocative for right-to-left shunting through a patent foramen ovale. Sleep. (2002) 25:856-62. doi: 10.1093/sleep/25.8.21

49. Lee MJ, Park S-J, Yoon CH, Hwang J-W, Ryoo S, Kim SJ, et al. Association of Left Atrial Enlargement with Cortical Infarction in Subjects with Patent Foramen Ovale. J Stroke. (2016) 18:304-11. doi: 10.5853/jos.2016. 00290

50. Lee J-D, Hu Y-H, Lee M, Huang Y-C, Kuo Y-W, Lee T-H. High risk of one-year stroke recurrence in patients with younger age and prior history of ischemic stroke. Curr Neurovasc Res. (2019) 16:250-7. doi: 10.2174/1567202616666190618164528

51. Friberg L, Rosenqvist M, Lip GYH. Evaluation of risk stratification schemes for ischaemic stroke and bleeding in 182678 patients with atrial fibrillation: the Swedish Atrial Fibrillation cohort study. Eur Heart J. (2012) 33:1500-10. doi: 10.1093/eurheartj/ehr488

Conflict of Interest: BM and KN received speaker's fees from Abbott. DT is a member of the RESPECT trial steering committee and received fees from Abbott.

The remaining authors declare that the research was conducted in the absence of any commercial or financial relationships that could be construed as a potential conflict of interest.

Copyright (c) 2020 Kahles, Michel, Hapfelmeier, Eberli, Zedde, Thijs, Kraemer, Engelter, Serena, Weimar, Mallmann, Luft, Hemelsoet, Thaler, Müller-Eichelberg, De Pauw, Sztajzel, Armon, Kent, Meier, Mattle, Fischer, Arnold, Mono and Nedeltchev. This is an open-access article distributed under the terms of the Creative Commons Attribution License (CC BY). The use, distribution or reproduction in other forums is permitted, provided the original author(s) and the copyright owner(s) are credited and that the original publication in this journal is cited, in accordance with accepted academic practice. No use, distribution or reproduction is permitted which does not comply with these terms. 